\title{
Uncharted waters: rare and unclassified cardiomyopathies characterized on cardiac magnetic resonance imaging
}

\author{
Ailbhe C. O'Neill • Shaunagh McDermott • \\ Carole A. Ridge • Kenneth McDonald • David Keane • \\ Jonathan D. Dodd
}

Received: 19 July 2010 /Accepted: 24 September 2010 /Published online: 19 October 2010

(C) European Society of Radiology 2010

\begin{abstract}
Cardiac magnetic resonance imaging (CMR) has undergone considerable technology advances in recent years, so that it is now entering into mainstream cardiac imaging practice. In particular, CMR is proving to be a valuable imaging tool in the detection, morphological assessment and functional assessment of cardiomyopathies. Although our understanding of this broad group of heart disorders continues to expand, it is an evolving group of entities, with the rarer cardiomyopathies remaining poorly understood or even unclassified. In this review, we describe the clinical and pathophysiological aspects of several of the rare/unclassified cardiomyopathies and their appearance on CMR.
\end{abstract}

Keywords Humans - Magnetic resonance imaging · Cardiomyopathies/diagnosis · Ventricular dysfunction/ classification

\section{Introduction}

The prevalence of cardiomyopathy in the United States is approximately one in 5,439 or $0.02 \%$ of the population [1]. Approximately 26,000 deaths per annum are attributed to cardiomyopathy, which follows coronary heart disease as the commonest cause of sudden death. Transthoracic echocardiography (TTE) is the mainstay imaging modality for

A. C. O’Neill · S. McDermott • C. A. Ridge · J. D. Dodd $(\triangle)$ Department of Radiology, St Vincent's University Hospital, Elm Park,

Dublin 4, Ireland

e-mail: jonniedodd@gmail.com

K. McDonald $\cdot$ D. Keane

Department of Cardiology, St Vincent's University Hospital, Elm Park,

Dublin 4, Ireland cardiomyopathy assessment [2]. Nevertheless, poor acoustic windows can make it difficult to optimally assess certain patients.

Several evolving technical developments have allowed cardiac magnetic resonance imaging (CMR) to enter mainstream cardiology imaging practice for cardiomyopathy assessment [3]. Increasing magnet strengths and surface coil channels and rapid k-space sampling have resulted in faster scan times, placing CMR in a primary position in the work-up of many complex cardiomyopathies [4]. Several consensus statements include CMR as a primary imaging technique in cardiomyopathy assessment $[3,5]$. In this review, we outline the clinical and pathophysiological aspects of several of the rarer or unclassified cardiomyopathies and their appearances on CMR.

\section{Classification of cardiomyopathy}

In 2006 a re-classification of the cardiomyopathies was published, as our understanding of the importance of genetics in the pathophysiology of the cardiomyopathies became more central [6]. This consensus report revised the definition of cardiomyopathy to include "mechanical or electrical dysfunction that usually exhibits inappropriate ventricular hypertrophy and dilatation due to a variety of causes that frequently are genetic". Under this classification system, the division of cardiomyopathy was divided into primary and secondary causes. A more recent classification published by Elliott et al. [7] from the European society of cardiology working group on myocardial and pericardial diseases places an emphasis on phenotypic classification.

In this system, cardiomyopathies are divided into five specific morphological and functional subgroups: hypertrophic, dilated, arrhythmogenic, restrictive and unclassified 


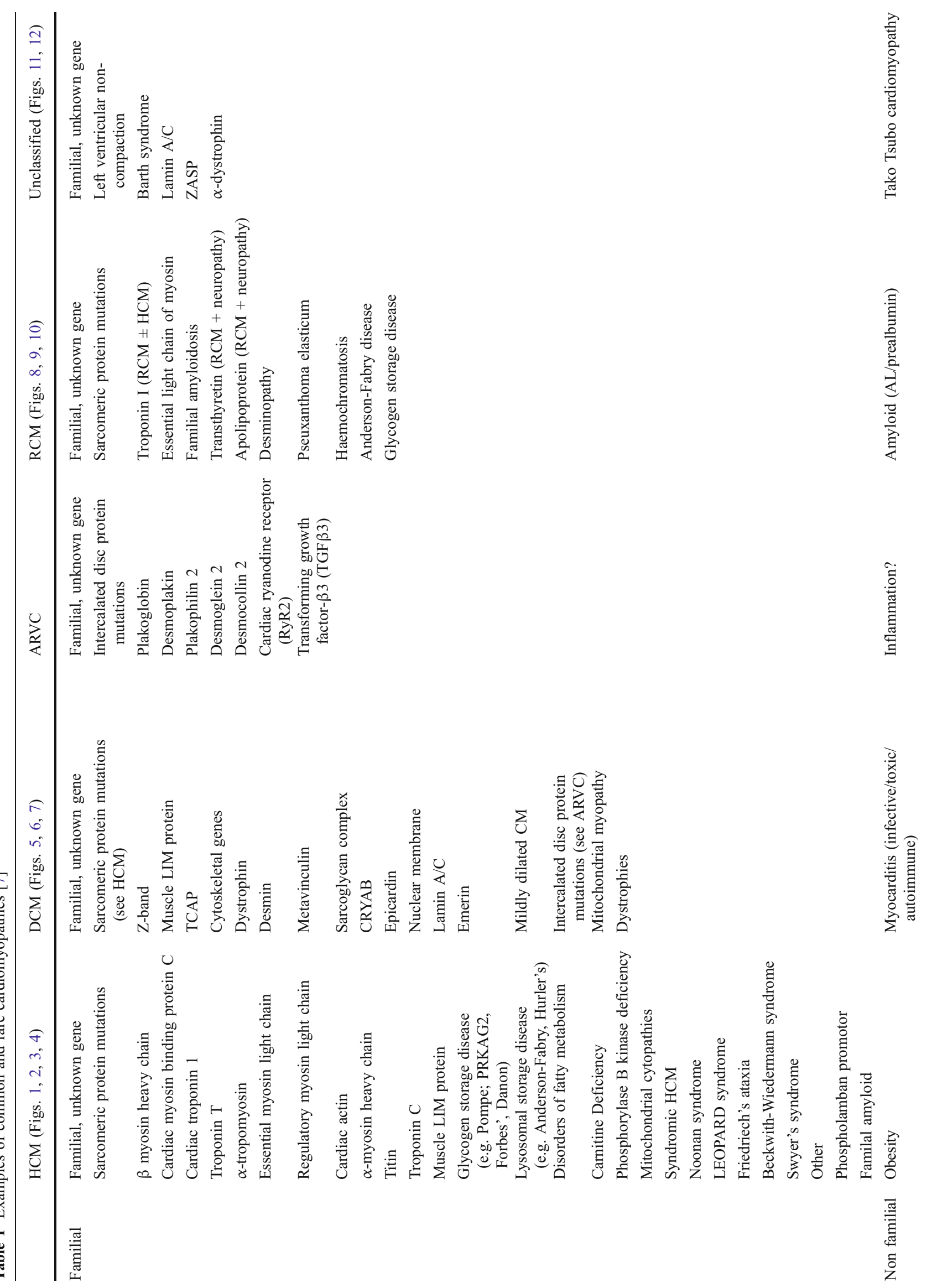




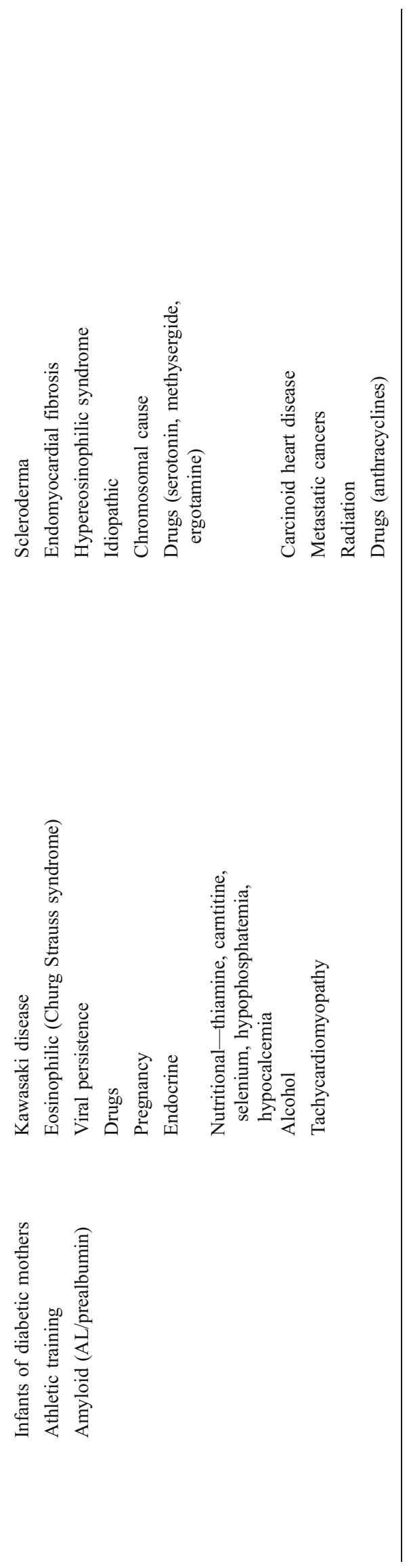

(Table 1). Each of these groups is then further subdivided into familial and non-familial forms. Each morphological group of cardiomyopathies has common and rare forms.

Uncommon cardiomyopathies characterized by hypertrophy

Hypertrophic cardiomyopathy (HCM) is defined by the presence of increased ventricular wall thickness or mass without a loading condition such as hypertension or valvular disease [7].

Cardiac amyloidosis

Cardiac amyloidosis describes amyloid deposition in the heart, which may occur as part of systemic amyloidosis or as a localized process.

Systemic amyloidosis (SAA) is a complication of chronic inflammatory conditions, with renal disease being the predominant feature, presenting with proteinuria and renal failure. Cardiac involvement is rare [8]. Systemic amyloidosis (SAL) is the most commonly diagnosed form of clinical amyloid. Multiorgan involvement is common and the heart is affected approximately $90 \%$ of the time. Diastolic heart failure with right heart failure is the most common mode of presentation [8]. Hereditary systemic amyloidosis is due to deposition of amyloid fibrils derived from transthyretin, lysosome or apolipoprotein A-1. Clinical syndromes include cardiomyopathy, nephropathy or neuropathy. Senile systemic amyloidosis is caused by deposition of amyloid fibrils derived from normal wild type transthyretin and presents as a slowly progressive infiltrative amyloid cardiomyopathy [8].

\section{CMR appearances}

Cardiac amyloid typically demonstrates a diffuse decrease in signal intensity on T1 weighted FSE images [9, 10]. It generally causes diffuse hypertrophy of both the left and right ventricles (Fig. 1a), in contradistinction to HCM, which typically causes more focal hypertrophy. Thickening of the interatrial septum (Fig. 1a) and posterior right atrial wall $>6 \mathrm{~mm}$ is also seen in cardiac amyloidosis [11]. Late gadolinium enhancement (LGE) is another hallmark of cardiac amyloidosis on CMR. In a study by Vogelsberg et al. [12], LGE was demonstrated in $79 \%$ of patients with cardiac amyloidosis. Several different patterns of LGE were seen. There was LGE of the entire subendocardial circumference, extending in various degrees into neighbouring myocardium (Fig. 1b). Late gadolinium enhancement was also distributed in large areas in the left ventricle, mainly involving subendocardial areas circumferentially. Subepicardial myocardium was typically less affected. Late gadolinium enhancement in the papillary muscles was also seen in approximately $40 \%$ of patients (Fig. 1b). Ejection 

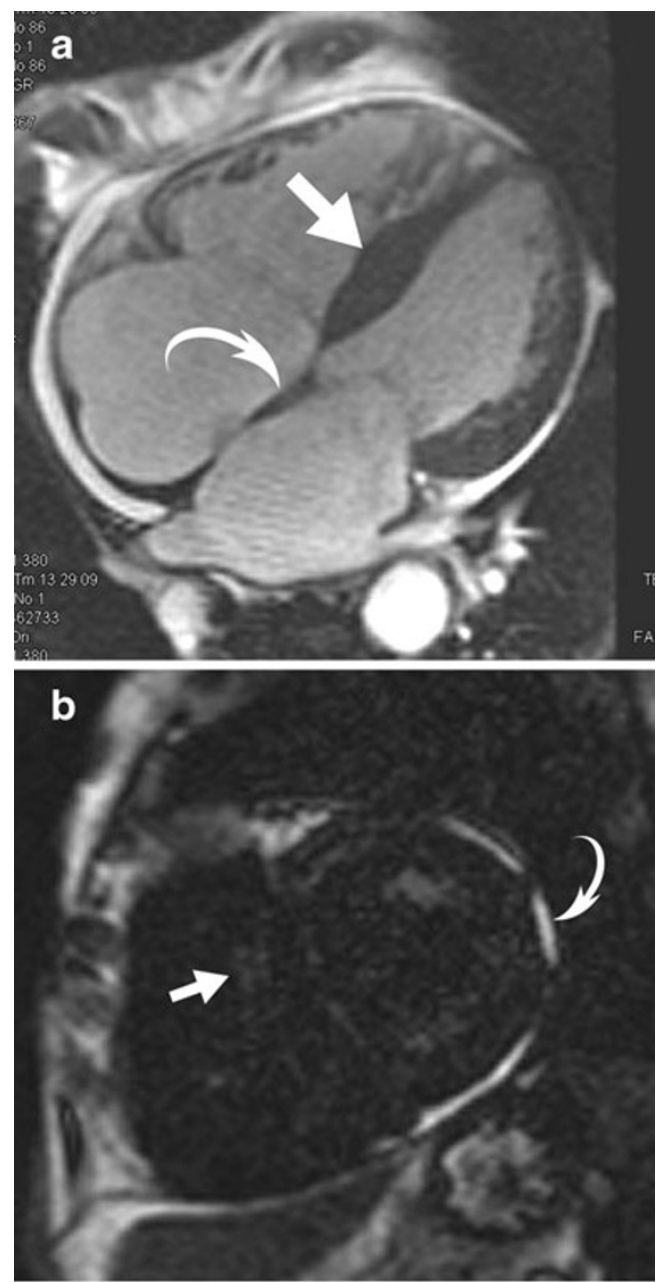

Fig. 1 A 78-year-old man who presented with progressive heart failure. RV endomyocardial biopsy showed cardiac amyloid. a Horizontal long axis SSFP sequence showed hypetrophy of the basal segments of the LV (straight arrow), biatrial enlargement and thickening of the interatrial septum (curved arrow). Note the small pericardial effusion. b Late-enhanced sequence showed circumferential subendocardial high signal. Note the high signal on the RV side of the interventricular septum resulting in the tram track sign (straight arrow). Note also the small pericardial effusion (curved arrow)

fractions, left ventricular end-diastolic volume and myocardial mass were not significantly different between the cardiac amyloid group and the other group of patients with various cardiac disorders. The average interventricular septum was $17 \pm 4 \mathrm{~mm}$ in the amyloid group compared with $13 \pm 3 \mathrm{~mm}$ in the non-amyloid group [12]. A more recent study by Syed et al. [13] of CMR appearances in 120 patients with cardiac amyloidosis demonstrated LGE in $97 \%$ of patients and increased left ventricular wall thickness in $91 \%$. Global transmural or subendocardial LGE was the most common pattern seen in $83 \%$ of patients and this was associated with greater interstitial amyloid deposition. In a study of CMR appearances in 16 patients with amyloidosis, a mild or moderate pericardial effusion was seen in $46 \%$ of patients and a pleural effusion in 50\% [14].
Friedreich's ataxia

Friedreich's ataxia is the most common of the hereditary ataxia syndromes. Clinical features are secondary to a reduction in fraxetin synthesis associated with an unstable expansion of a GAA trinucleotide repeat [15]. Patients are homozygous when a GAA expansion is present on two alleles, irrespective of the length of the expansion, and heterozygous when a GAA expansion is found on one allele and a point mutation on the other allele [16]. The clinical hallmark is progressive ataxia, generally starting before 25 years of age, as well as dysarthria, sensory neuropathy, lower limb areflexia, extensor plantar response and weakness from degeneration of dorsal columns and pyramidal tracts [17]. Skeletal abnormalities and glucose intolerance are also present to a variable degree. Cardiac involvement in Friedreich's ataxia is a common finding and is characterized by increased thickness of the ventricular wall that is typically concentric and symmetrical, with a normal or small LV cavity and normal systolic function [18]. The age of disease onset and the rate of clinical progression are variable and are influenced by the size of the GAA expansion in the smaller allele (GAA1) [19].

\section{CMR appearances}

Rajagopalan et al. [19] demonstrated a correlation between increasing GAA1 repeat numbers and increasing LV mass (Fig. 2a, b). Left ventricular mass was higher in patients with larger GAA1 (>600) repeat sizes. Left ventricle mass positively correlated with the genetic severity of the disease and is seen in earlier onset disease and short disease duration. Longer disease duration is associated with smaller LV mass. Septal and posterior wall thickness showed similar changes with greater thickness seen in patient groups with $>600$ GAA1 repeats [19]. The number of patients with severe hypertrophy was relatively small and overt hypertrophy relatively greater in patients with larger GAA1 repeats $[18,20]$.

Noonan syndrome

Noonan syndrome is characterized by short stature, typical facies and congenital heart defects. Noonan syndrome occurs in an autosomal dominant or sporadic inheritance. Male and females are equally affected [21]. Its pathophysiology is not fully understood. Four disease-causing genes have been identified which are involved in the RAS/RAF/MEK/ERK signal transduction pathway. These are PTPN11, SOS1, RAF1 and KRAS [22, 23]. The cardinal features of Noonan syndrome are unusual facies including hypertelorism, down-slanting eyes, epicanthic folds, low-set posterior ears, micrognathia and a webbed neck [24]. Short stature, chest 

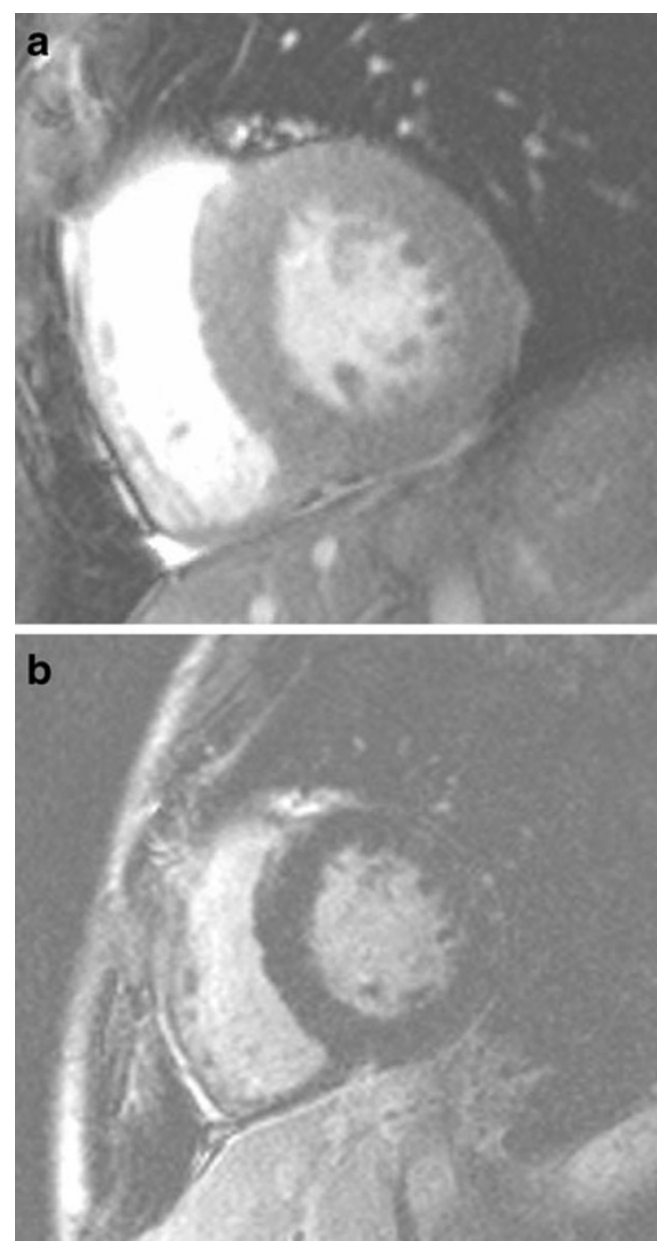

Fig. 2 A 19-year-old man with Friedrichs ataxia who presented with acute chest pain. a Short-axis SSFP sequence showed circumferential hypertrophy of the left ventricle. b Late-enhanced sequence showed an absence of high signal. Late-enhancement has not been described in FA

deformity, bleeding diathesis and mental retardation are also cardinal features [25]. The phenotype becomes less pronounced with increasing age [21].

\section{CMR appearances}

The most common cardiac defect is pulmonary valve stenosis, which affects approximately $50 \%$ of patients with Noonan syndrome [26]. Pulmonary stenosis is more common in patients with PTPN11 mutations. Atrial septal defects occur in approximately $10 \%$, persistent ductus arteriosus in 3\% and ventricular septal defects in 5\% [21]. Asymmetric septal hypertrophy is present in $20 \%$ of patients (Fig. 3a, b) [27, 28]. A case report by Hudsmith et al. [28] described the CMR appearances in a woman with Noonan syndrome. The patient underwent open pulmonary valvotomy and secundum atrial septal defect closure as a child. There was asymmetrical septal hypertrophy detected on CMR with an increased LV mass. Following gadolinium, there was patchy increased signal in the anterior, anteroseptal and lateral walls. Myocardial hypertrophy associated with Noonan syndrome is not strongly associated with sudden death or rhythm disturbances though it is histologically similar to non-syndromic HCM [26].

\section{Danon disease}

Danon disease is a multisystemic, lysosomal, glycogenstorage disease with normal acid maltase [29]. It is characterized by a triad of cardiomyopathy, skeletal myopathy and variable mental retardation with intracytoplasmic vacuoles containing autophagic material and glycogen in cardiac and skeletal muscle cells [30, 31]. Danon disease is an X-linked dominant disorder, though spontaneous mutations have also been reported. Males are
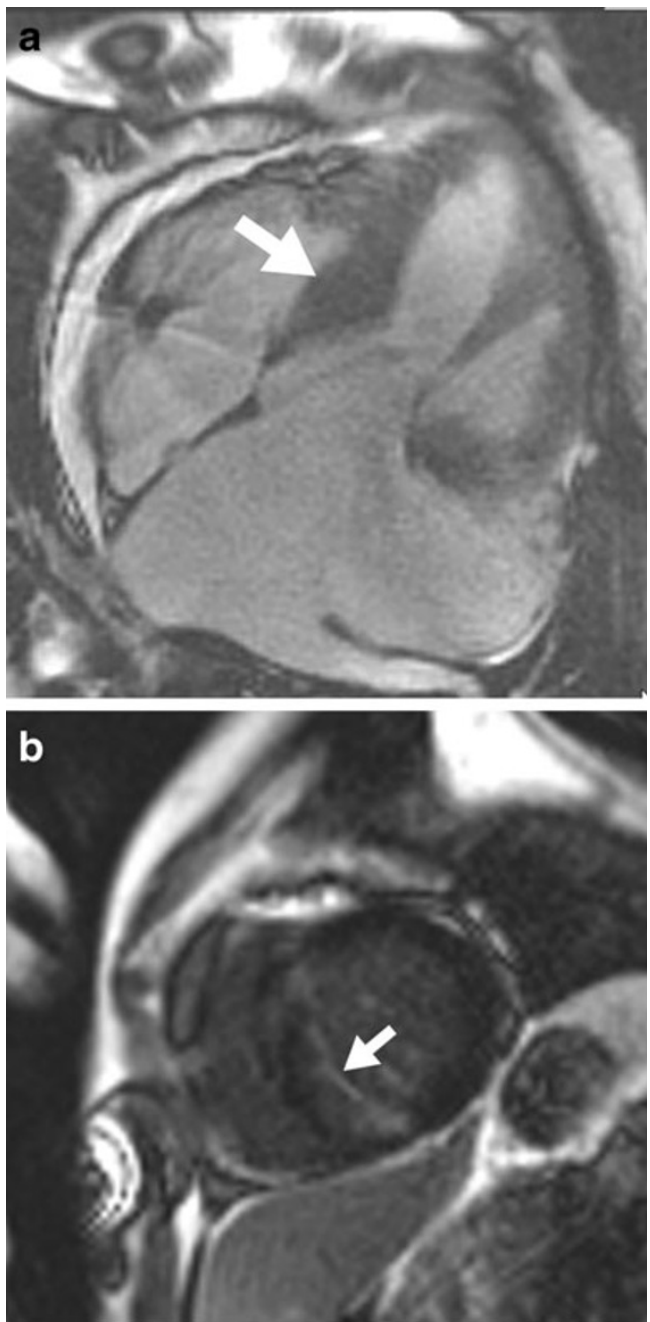

Fig. 3 A 46-year-old man with known Noonan's syndrome. a Horizontal long axis SSFP sequence showed septal hypertrophy (arrow) and a dilated left atrium secondary to mitral regurgitation. b Late-enhanced short axis sequence showed extensive high signal involving the antero- and infero-septal myocardial segments (arrow) 
usually more severely affected than females and also present at an earlier age. Age of presentation in males ranges from infancy to the second decade and affected males rarely survive beyond their $20 \mathrm{~s}$ and die secondary to an arrhythmia or congestive cardiac failure [29]. Female carriers develop cardiomyopathy during adulthood, most commonly in the 4 th or 5 th decades. Both skeletal myopathy and mental retardation are less common in females [30]. Wolff-Parkinson-White, increased serum creatinine kinase and ophthalmic abnormalities are also seen in Danon disease [31, 32]. Mutations in the lysosomeassociated protein 2 (LAMP-2) have been identified as the causative gene [31]. LAMP-2 is a heavily glycosated protein found inside the lysosomal membrane. Its deficiency results in excessive myocardial glycogen accumulation, which is responsible for the myocardial hypertrophy [30].

\section{CMR appearances}

The glycogen storage disorders generally induce myocardial hypertrophy. CMR is useful for assessing the severity of hypertrophy and assessing function. Either concentric or asymmetric hypertrophy is present in most male patients, although some present with late-stage dilated cardiomyopathy (Fig. 4a, b). Female patients usually present with dilated cardiomyopathy [33].

Piotrowska-Kownacka et al. [34] describe the CMR appearances of a patient with Danon disease. Subendocardial perfusion defects were visible in almost all segments on first-pass perfusion sequences. These were located predominantly in the lateral and anterior walls. Late gadolinium enhancement was seen in the subendocardium in the septal segments and transmurally in the anterior and lateral walls.

Uncommon cardiomyopathies characterized by dilation

Dilated cardiomyopathies (DCM) are characterized by dilatation of the cardiac chambers with impaired contraction of the ventricles. DCM in the familial from is usually related to cytoskeletal genetic mutations. Non-familial causes are numerous and include myocarditis and drug causes.

\section{Peripartum cardiomyopathy}

Peripartum cardiomyopathy (PPCM) is a rare cause of heart failure. It typically develops in the final month of pregnancy or within 5 months of delivery. Its aetiology appears multifactorial and complex. Several proposed theories for its aetiology exist, including a form of viral myocarditis, an abnormal immune response to pregnancy, an abnormal response to hameodynamic stress of pregnancy, increased myocyte apoptosis, cytokine-mediated myocardial inflam-
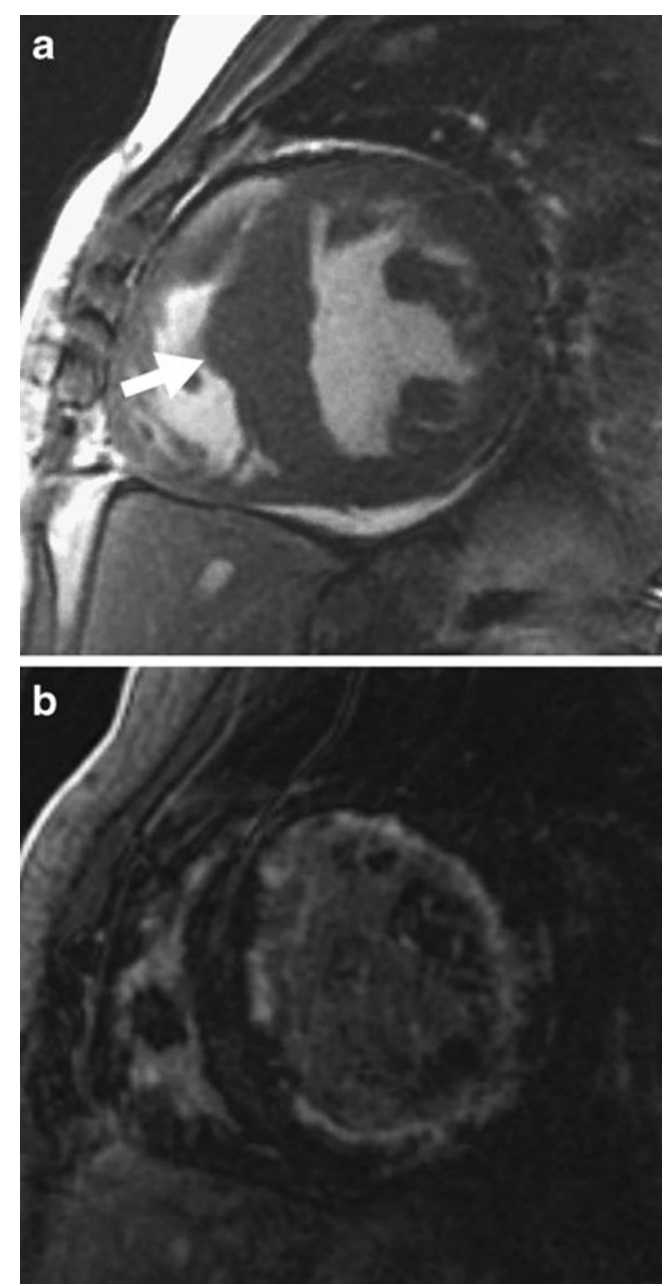

Fig. 4 A 21-year-old man with known Danon's glycogen storage disorder. a Short-axis SSFP sequence showed hypertrophy of the interventricular septum (arrow). b Late-enhanced short axis sequence showed circumferential high signal in the LV

mation, malnutrition, genetic factors, excessive prolactin production, abnormal hormone function and increased adrenergic tone [35].

\section{CMR appearances}

Reports of the CMR appearances in PPCM show conflicting results. Generally, patients present with a dilated left ventricle (Fig. 5a, b). Results following the administration of gadolinium are conflicting. Kawano et al. [36] described a case of peripartum cardiomyopathy in which CMR at 2 months demonstrated diffuse epicardial and mid-wall late gadolinium enhancement in the left ventricle. The late gadolinium enhancement decreased on follow up CMR performed at 10 months. Caballero-Borrego et al. [37] described a case of PPCM imaged with CMR in the chronic phase. CMR demonstrated extensive linear LGE at the septal segments. In contrast, a case report by Leurent et al. [38] in a patient with PPCM failed to show any LGE on 

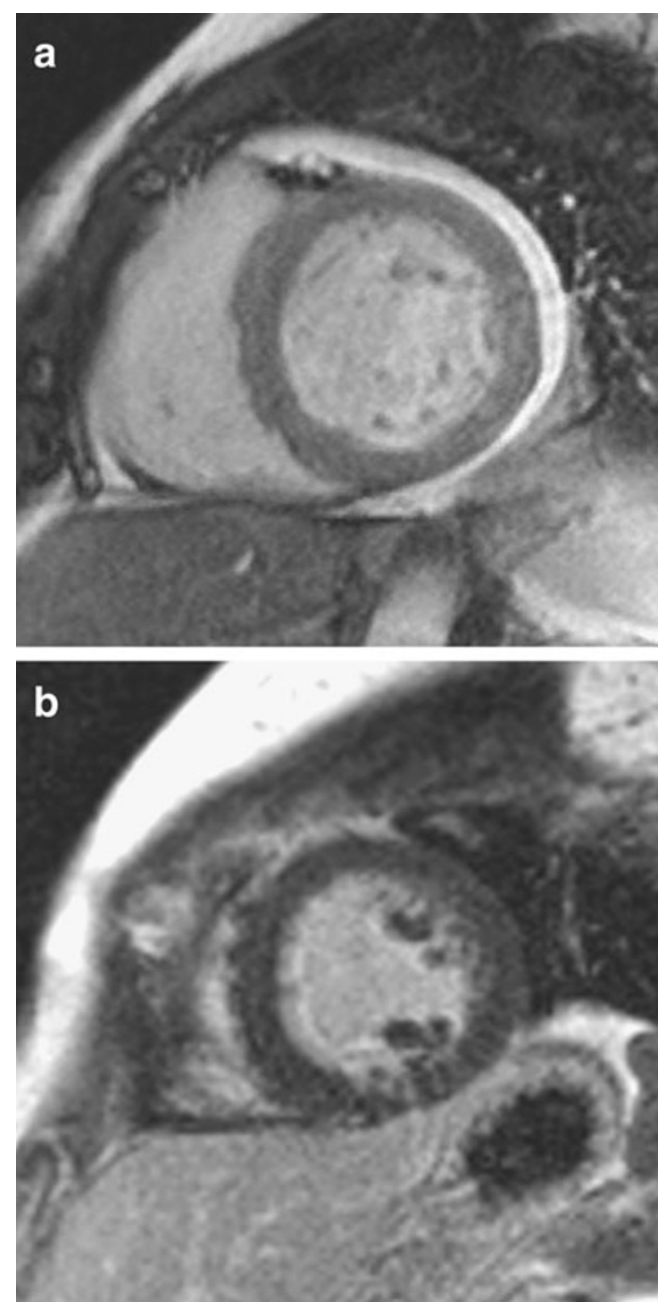

Fig. 5 A 34-year-old woman with progressive shortness of breath 1 week following post-partum. a Short-axis SSFP sequence showed mild dilation of the left ventricle (end-diastolic diameter $=62 \mathrm{~mm}$ ). $\mathbf{b}$ Late-enhanced sequence showed an absence of high signal in this case

CMR 8 days after the onset of symptoms. A series of eight patients by Mouquet et al. [39] evaluating the CMR appearances in PPCM also failed to demonstrate myocardial LGE, even in patients imaged within 2 weeks of symptoms. No specific CMR characteristics were demonstrated between the patients who regained LV function and those who did not. Marmursztein et al. [40] described two patients with PPCM imaged with CMR. One patient demonstrated no CMR abnormality. The second patient had several areas of myocardial LGE. At clinical follow-up, the patient with a normal CMR had full recovery of cardiac function and was asymptomatic. The patient with LGE on CMR had persistent LV dysfunction.

This disparity in CMR reports is likely to be related to the numerous causes of peripartum cardiomyopathy. Baruteau et al. [41] divided peripartum cardiomyopathy into two distinct subgroups: (1) non-inflammatory cardiomyopathy secondary to malnutrition, genetics, excessive prolactin production, abnormal hormone function and increased adrenergic tone. The non-inflammatory cardiomyopathies do not appear to cause late gadolinium enhancement or T2 hypersignal; (2) inflammatory cardiomyopathy are secondary to viral myocarditis, abnormal immune responses in pregnancy, abnormal response to hemodynamic stress, increased myocyte apoptosis and cytokine-mediated inflammation. Inflammatory causes do appear to cause late gadolinium enhancement on CMR.

\section{Muscular dystrophy}

Duchenne (DMD) and Becker (BMD) muscular dystrophies are $\mathrm{X}$-linked genetic disorders due to mutations in the dystrophin gene. The protein dystrophin is totally absent or dysfunctional in DMD and is reduced in expression or mildly dysfunctional in BMD. Duchenne muscular dystrophy is a more severe disorder and patients die of respiratory failure, generally surviving only until the 3rd decade. In contrast, BMD has a slower decline and patients may survive until the 6th decade. Cardiomyopathy is the main cause of death in BMD [42].

The incidence of cardiomyopathy in DMD is zero under the age of 10 years, one-third at 14 years, half at 18 years and $98 \%$ over 18 years [43].

\section{CMR appearances}

Early cardiac evaluation of patients with BMD and DMD is required to assess those with early signs of cardiac abnormalities who may benefit from early intervention. Cardiac involvement in BMD begins at the subepicardium of the inferolateral wall in the third decade of life and increases in extent with age. Progressive myocardial damage causes regional loss of contractility and a progressive decrease in LV systolic function (Fig. 6a). Yilmaz et al. [44] studied 15 patients with BMD. Twelve patients had evidence of cardiac involvement by CMR. Patients with reduced left ventricular ejection fraction were older, with heavier hearts and regional wall motion abnormalities. Late gadolinium enhancement was seen on CMR in DMD involving predominantly the inferolateral free wall, the basal inferior and anterolateral region of the left ventricle (Fig. 6b). There was sparing of the right ventricle and interventricular septum $[45,46]$. Cardiac involvement in BMD is uncommon under the age of 16 years, increasing to approximately $70 \%$ by 40 years of age $[44,47]$.

Drug related dilated cardiomyopathies

Myocarditis is an inflammatory disease of the myocardium with a wide range of clinical presentations. It may be 

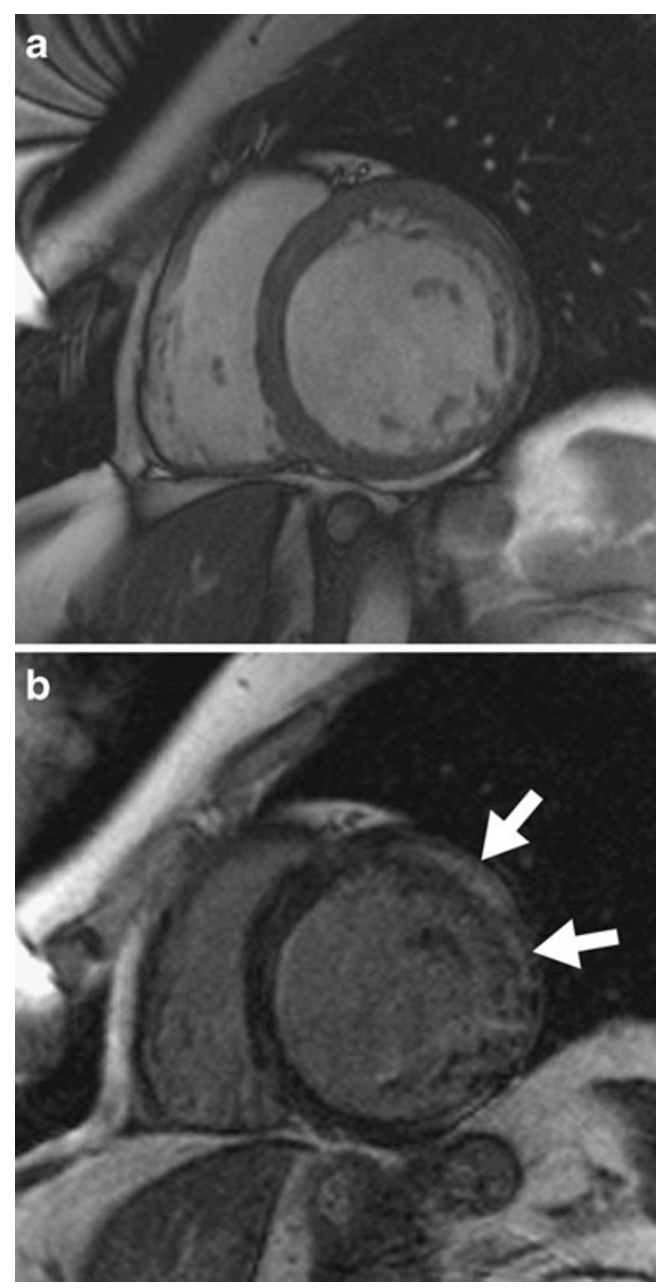

Fig. 6 A 46-year-old man with progressive heart failure and known Becker's muscular dystrophy. a Short-axis SSFP sequence showed dilation of the left ventricle (end-diastolic diameter $=70 \mathrm{~mm}$ ). b Lateenhanced sequence showed extensive transmural high signal throughout the lateral segments (arrows)

caused by a wide variety of infectious organisms, autoimmune disorders and drugs. It typically manifests in a previously healthy person and may result in rapidly progressive heart failure and arrhythmia. Acute myocarditis with established ventricular dysfunction can progress to dilated cardiomyopathy [48].

There are several case reports of ephedra-associated myocarditis [49-51]. Ephedra is a potent sympathomimetic agent with direct and indirect effects on adrenergic receptors. An adverse effect of adrenergic stimulation is myocyte toxicity [52]. Naik et al. [52] described two men, aged 19 and 21 years respectively, who were admitted on separate occasions with dilated cardiomyopathy secondary to ephedra. Methamphetamine is a sympathomimetic agent that causes sympathetic stimulation by excessive release of noradrenaline and blockade of re-uptake at the sympathetic synaptic receptors. Several case reports have documented reversible dilated cardiomyopathy secondary to methamphetamine use [53, 54]. A case report by Lopez et al. [55] evaluated the CMR appearances in a patient presenting with cardiomyopathy secondary to methamphetamine. CMR demonstrated a reduced ejection fraction of $37 \%$ but no evidence of LGE, indicating no acute myocardial inflammation. The patient improved following treatment and repeat CMR showed improved ejection fraction to $64 \%$. In our institution, we have recently encountered a patient with mephedrone-associated myocarditis. Mephedrone is a cathinone derivative that is purchasable legally on the Internet or in 'head-shops'. This substance bears similar pharmacological and structural similarities to amphetamine. This is also referred to as 'meow', 'meph', 'rush' and 'roxy'. People presenting to hospital after taking mephedrone describe a number of cardiovascular signs, agitation and sometimes a drug-induced psychosis [56]. At least four deaths in the UK in March 2010 and a further 18 deaths in England and seven in Scotland have been attributed to cathiniones [57].

\section{CMR appearances}

Drug-induced myocarditis CMR typically shows high signal on T2-weighted sequences, an increased global enhancement ratio and high signal on delayed enhanced sequences (Fig. 7a and b). There is often regional wall motion hypokinesis in the lateral segments. The abnormal enhancement typically affects the lateral segments.

Uncommon cardiomyopathies characterized by restriction

Restrictive cardiomyopathies demsontrate ventricular diastolic dysfunction, and typically bilateral atrial enlargement with relatively normal ventricular end-diastolic volume and systolic function.

\section{Systemic sclerosis}

Systemic sclerosis (SSc) is a connective tissue disorder of unknown aetiology characterized by diffuse vascular lesions, fibrosis of the skin and other organs, including the heart, kidneys and lungs. Excess collagen deposition is a characteristic feature of systemic sclerosis. Systemic sclerosis is divided into five forms: diffuse, limited, transitory, systemic scleroderma sine scleroderma and malignant scleroderma. The principal forms are diffuse and limited systemic scleroses [58]. Diffuse systemic sclerosis is characterised by Raynaud phenomenon that precedes the development of skin changes by one year [59]. There is generalised skin fibrosis of the chest and limbs, skin hyperpigmentation or hypopigmentation There is early involvement of the lungs, kidneys, gastrointestinal tract and 

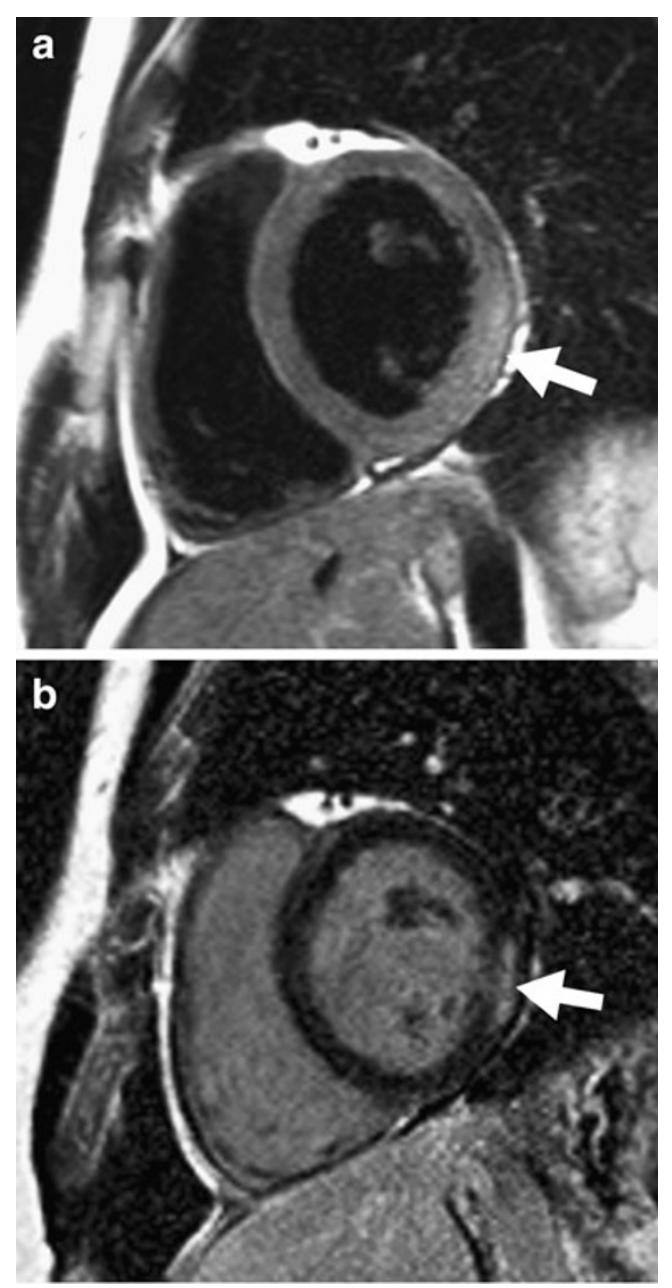

Fig. 7 A 21-year-old man admitted with severe central chest pain 12 $\mathrm{h}$ after orally ingesting mephedrone. a T2-weighted sequence showed high signal in the lateral segments consistent with myocardial edema (arrow). b Late-enhanced short-axis sequence showed high signal in the mid-wall lateral segment consistent with acute myocardial inflammation (arrow)

the heart. Limited systemic slcerosis is characterized by sclerotic changes of the hands, feet, face and forearms. There is telangictasia of the skin, cutaneous calcification and late involvement of the lungs with late development of pulmonary hypertension. Diffuse systemic sclerosis occurs equally in males and females. Limited systemic sclerosis has a female predominance. Sytemic scleroderma sine scleroderma is characterised by involvement of the internal organs alone with no external signs [58]. Diastolic dysfunction may occur before any clinical sign or symptom of the disease, in both diffuse and limited forms of the disorder $[60,61]$.

\section{CMR appeareances}

Tzelepis et al. [62] reviewed the CMR appearances of 41 patients with SSc; 24 of $36(66 \%)$ demonstrated LGE characteristically midwall LGE with sparing of the subendocardium (Fig. 8). There was a linear pattern in all patients that was infrequently interrupted or spiculated. The LGE predominantly involved the basal and mid ventricular LV segments with less common involvement of the apices. Patchy nodular enhancement was seen in the lower or upper right ventricular insertion points in $17 \%$ of patients. Myocardial fibrosis appears to progress from base to apex. All patients with LGE had involvement of the basal segments. Midcavity involvement was always associated with basal involvement and apical involvement was associated with basal and midcavity fibrosis. Apical segment was seen in patients with a greater duration of Raynaud's phenomenon. Myocardial fibrosis was similar between diffuse and localized SSc and was more severe in patients with abnormal Holter results [62].

Hypereosinophilic syndrome

Idiopathic hypereosinophilic syndrome (HES) is characterized by peripheral eosinophilia and multiorgan dysfunction in the absence of known causes of hypereosinophilia. The heart is frequently involved and there are three stages of eosinophiliarelated cardiac damage. Stage one is an acute necrotic stage, which is usually clinically silent. An intermediate stage follows that is characterized by thrombus formation. This leads to the third stage, which is a fibrotic stage resulting in endomyocardial fibrosis and restrictive cardiomyopathy [63].

\section{CMR appearances}

Several case reports have documented the CMR appearances of HES (Fig. 9a-c) [64-67]. Syed el al. [67] describe

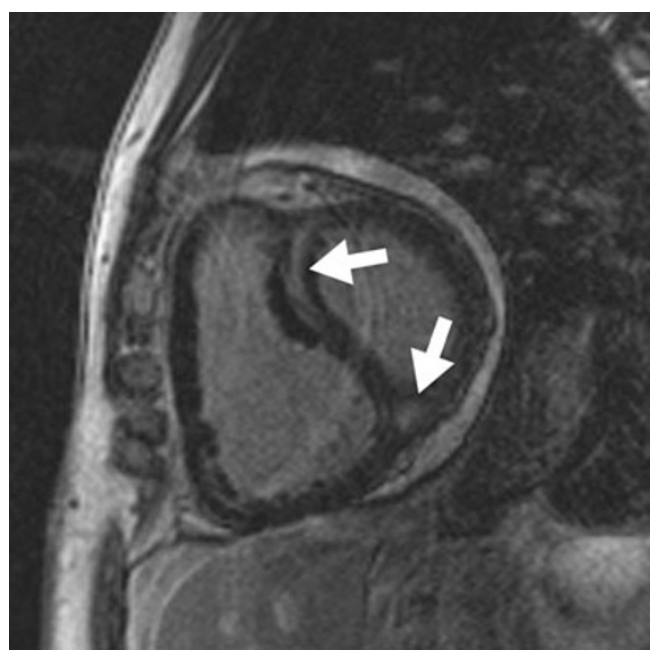

Fig. 8 A 55-year-old man who presented with progressive shortness of breath. He had a background history of severe systemic sclerosis. Late-enhanced short-axis sequence showed extensive high signal in the interventricular septum (arrows) 


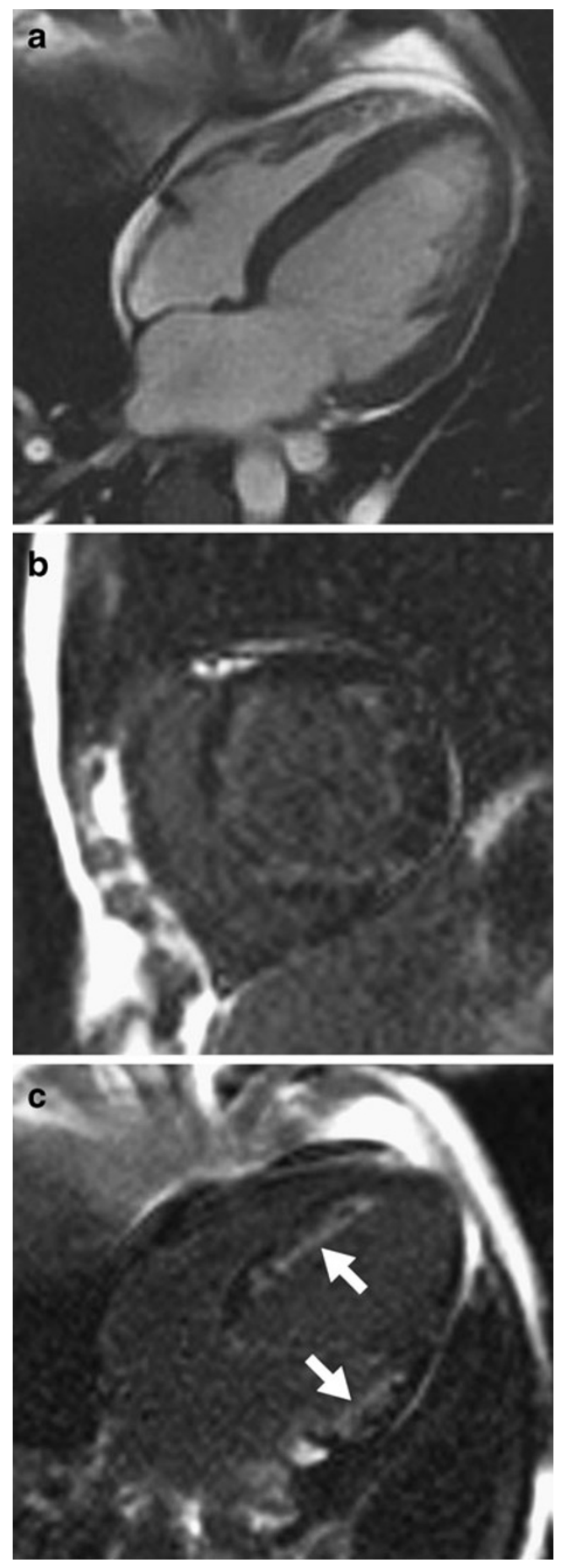

Fig. 9 A 38-year-old man with hypereosinophilic syndrome presented with progressive shortness of breath. Serum measurements showed a white cell count of $45,000,41,000$ of which were eosinophils. a Horizontal long-axis SSFP sequence showed mild circumferential hypertrophy of the left ventricle. b Late-enhanced short-axis sequence showed extensive high signal in the interventricular septum and lateral wall. c Late-enhanced horizontal long-axis view confirms extensive high signal throughout the septum and lateral basal segments (arrows) extensive thickening of the LV myocardium and partial obliteration of the LV cavity on systole. Following contrast, a three-layered appearance on CMR was identified, consisting of the normal uninfiltrated myocardium, a hyperintense subendocardium and a linear hypointense layer of thrombus found inside the endocardium. Similar hyperenhancement of the endocardium compared with the myocardium was seen on CMR by Salanitri [65].

\section{Metastatic disease}

Metastatic disease to the heart and pericardium is rare but is more common than primary cardiac tumours [68]. Cardiac metastases are associated with a poor prognosis. The most common tumours to metastasize to the cardiac structures are lung, lymphoma, breast, esophagus and melanoma [69, 70]. Bronchogenic carcinoma is the most common malignancy to spread to the cardiac structures and adenocarcinoma is the most common histological type [70]. Metastatic disease from bronchogenic carcinoma to the heart may be via direct spread, lymphatic or haematogenous spread. Intrapericardial extension of tumour into the pericardium and left atrium should be suspected when there is obliteration of the superior pulmonary vein [71].

Involvement of the heart and pericardium is usually a late manifestation of lymphoma. Cardiac involvement occurs in $16 \%$ of patients with Hodgkin's disease and $18 \%$ of patients with non-Hodgkin lymphoma. Metastases are usually firm, focal nodules, which can be found in the walls of all cardiac chambers and in the pericardium [72]. Breast cancer may spread via direct extension. Enhancement of the pericardium or pericardial nodularity is suggestive but not definitive of malignant involvement [73].

In melanoma, autopsy reports have shown myocardial tissue replaced by extensive nodular deposits appearing as pigmented epicardial 'black' implants. This black macroscopic appearance gave rise to the term 'charcoal heart' [74].

\section{CMR appearances}

Most cardiac tumours are low signal intensity on T1 sequences and brighter on T2 sequences (Fig. 10a, b) [75]. Malignant disease enhances post contrast administration (Fig. 10c). A case reported by Rathi et al. [76] described the CMR appearances of 'charcoal heart'. There was nodularity of the LV myocardium with varying penetration into the endocardium and epicardium. On SSFP cine sequences, the nodular deposits were isointense to bright when compared with normal myocardium. There were also multiple mass-like deposits lining the right atrial and left atrial wall with similar imaging signal characteristics to the LV myocardium. These nodular deposits were high signal on both $\mathrm{T} 1$ and $\mathrm{T} 2$ sequences. Masses 

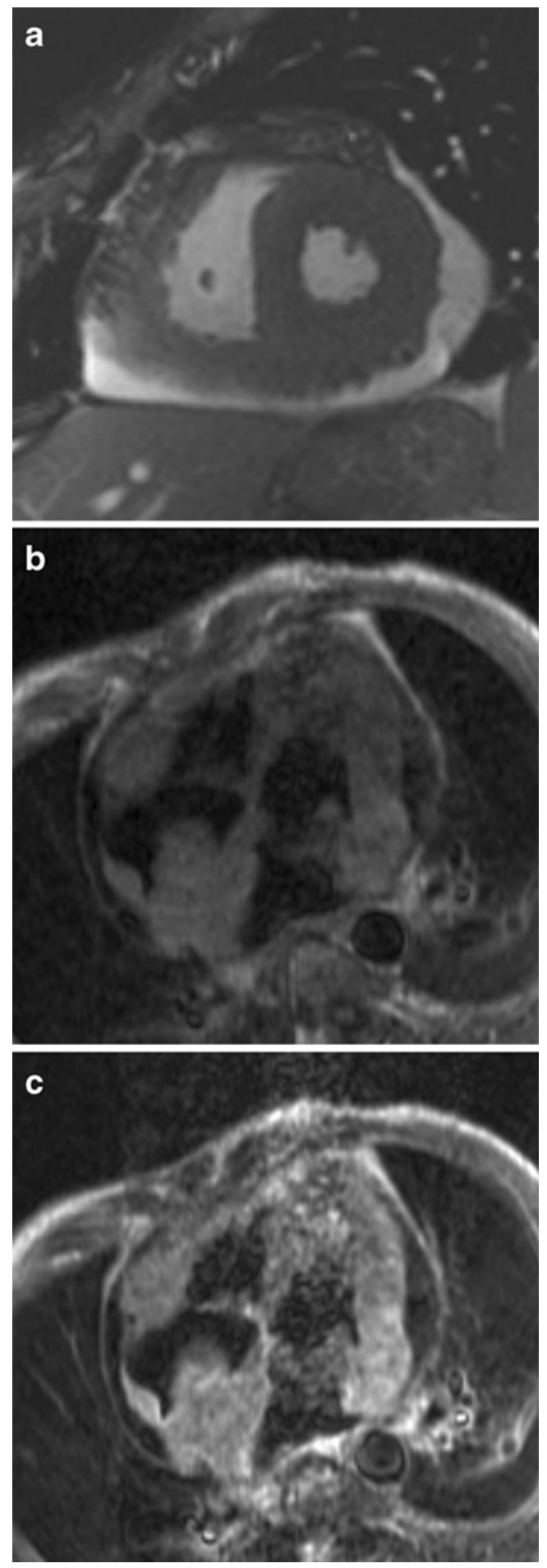

Fig. 10 A 46-year-old man with progressive shortness of breath. He was diagnosed with HIV and endomyocardial biopsy revealed cardiac lymphoma. Echocardiography suggested diastolic heart failure. a Short-axis SSFP sequence showed circumferential infiltration of the basal segments of the right and left ventricle. b Pre-contrast T1-weighted horizontal long-axis sequence showed extensive lymphomatous infiltration of all four heart chambers. c Post-contrast T1-weighted horizontal long-axis sequence showed marked enhancement of the extensive lymphomatous infiltrating masses throughout the heart with high melanin content are hyperintense on $\mathrm{T} 1$ sequences due to shortening of the T1 relaxation time. Hyperintensity on $\mathrm{T} 2$ reflects high increased proton density or water content of the tumour. A case report by Deetjen et al. [77] described a cardiac metastasis from a renal cell adenocarcinoma, which also demonstrated a nodular enhancing mass. There was heterogeneous signal in the nodular mass on T1 and T2 sequences. Osteogenic sarcoma involving the heart is rare but merits mention as the metastasis contains bone. These calcific areas of increased opacity may be visible on chest $\mathrm{X}$-ray but are better characterized on CT. Calcification is shown as a signal void on CMR.

\section{Cardiomyopathies that are unclassified}

This group of unclassified cardiomyopathies includes left ventricular noncompation and Takotsubo cardiomyopathy.

\section{Left ventricular non-compaction}

Left ventricular non-compaction (LVNC) is a myocardial disorder characterized by prominent and excessive trabeculations and deep intratrabecular recesses. These recesses communicate with the ventricular cavity rather than the coronary circulation [78]. Chin et al. [79] were the first group to use the term LVNC in their review of eight cases in 1990. Left ventricular non-compaction is postulated to arise from an abnormality of the normal myocardial maturation process. The early fetal myocardium has deep recesses between loosely interwoven fibres, which communicate with the ventricular cavity. The trabeculations provide oxygenation exchange by creating a larger surface for direct exchange diffusion from the cardiac lumen. In the 5th-8th weeks of fetal life, the coronary circulation develops. obviating the need for direct exchange transfusion and in a normal fetus the loosely interwoven fibres undergo compaction [80]. The severity of LVNC will depend on the timing of the arrest in the myocardial maturation and compaction process. Compaction moves from base to apex, from epicardium to endocardium and from the septal to the lateral wall. This process of maturation explains why the apex and midventricular lateral segments are more commonly involved in LVNC [81]. Histology demonstrates that ventricular endothelium lines the recesses and is in continuity with the ventricular cavity [82]. Other histopathological characteristics include focal ischemic necrosis within the trabeculations and endocardial layer, compensatory hypertrophy of the myocardium, interstitial fibrosis and scarring reflecting focal replacement fibrosis [83].

Familial LVNC can be transmitted as an autosomal dominant trait with incomplete penetrance and less commonly as an autosomal recessive trait and X-linked inheritance are 
seen. Genetic mutations in the G4.5 and $\alpha$-dystrobrevin are seen in a minority of patients with LVNC [80, 84]. Noncompaction has been reported in association with neuromuscular disorders including Barth syndrome (also G4.5 genetic mutation), muscular and myotonic dystrophies, Pompe's disease, mitochondrial and various other genetic disorders [85]. It is also seen in association with other congenital cardiac defects, ventricular septal defects being the most frequent. Further congenital defects include subaortic obstruction, bicuspid aortic valves, coarctation of the aorta, Ebstein's anomaly, valvular pulmonary stenosis, Tetralogy of Fallot and pulmonary atresia $[86,87]$.

LVNC can present as fetal hydrops, neonatal heart failure and with ventricular fibrillation in its most severe form [80]. Cardiac failure is the most prevalent finding and complication in all studies of LVNC [78, 88]. Arrhythmias and thromboembolic events are more common in the adult population. In the study by Oechslin et al. [78], 11 thromboembolic events were seen in eight patients (24\%), one stroke, six episodes of transient ischaemic attacks, one mesenteric infarction and three episodes of pulmonary embolism. Ventricular tachycardia were seen in 14 patients (41\%).

\section{CMR appearances}

Echocardiography has previously been the mainstay imaging modality in the diagnosis of LVNC. Diagnostic criteria for LVNC on echocardiography have become confusing, some studies suggesting using a two layered myocardium with a non-compacted to compacted ratio of more than 2:1 (end systolic value) and colour Doppler flow within the trabeculations [89]. Other studies suggest using more than two thickened trabeculations as a diagnostic criteria.

CMR is increasingly performed for confirmation of the diagnosis and also to evaluate for complications related to the disorder. It has the advantage over echocardiography of optimal apical and lateral wall visualization, which are the most commonly involved segments [90]. Appearances can be variable with three basic features described by Dursun et al. [89]; extensive spongiform transformation of the left ventricular myocardium, trabeculations of the ventricular wall with deep recesses of the ventricular cavity and a dysplastic appearance of the myocardium with thinned myocardium and excessive trabeculations.

The end diastolic non-compacted to compacted ratio $(\mathrm{NC} / \mathrm{C})$ is higher in patients with LVNC (Fig. 11a). A compaction ratio can be quantitatively assessed by measuring thickness in millimeters of the noncompacted to the compacted myocardium. This can be done on a myocardial segmental basis using the standard 17-segment cardiac AHA model [91]. The $\mathrm{NC} / \mathrm{C}$ parameter is able to distinguish pathological noncompaction from lesser forms of non-
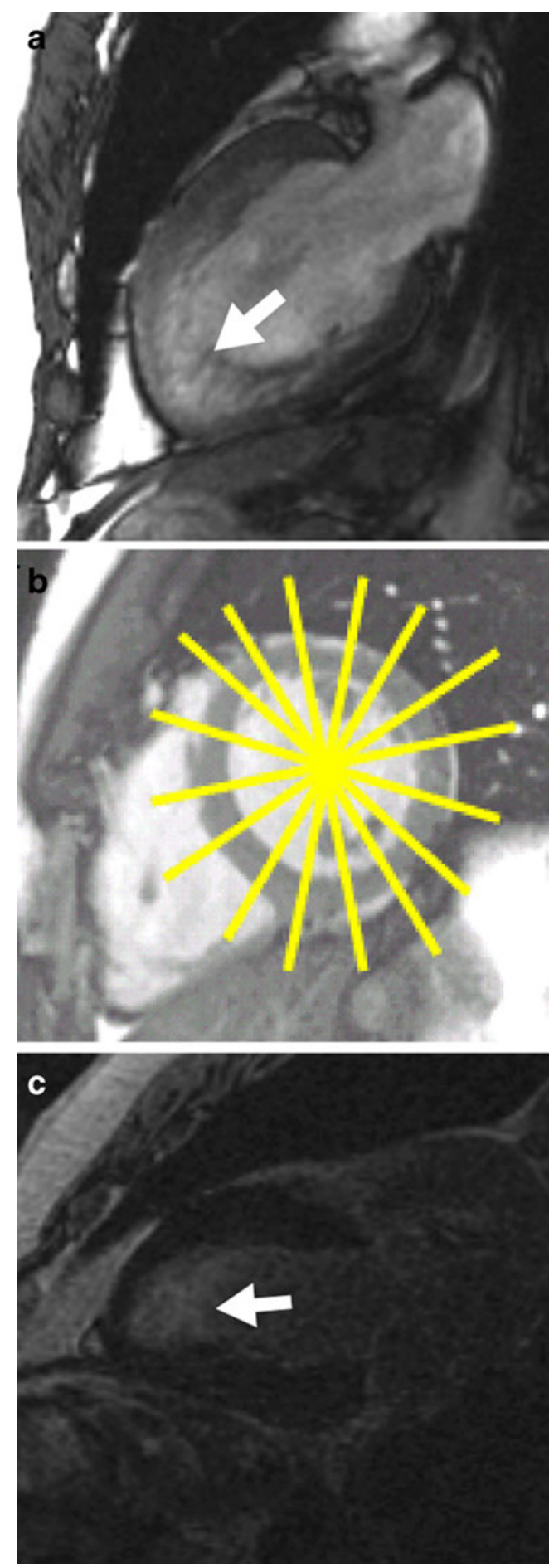

Fig. 11 A 38-year-old woman with progressive shortness of breath. a Vertical long-axis SSFP sequence showed increased trabeculations at the apical ventricular level. (arrow) b Short-axis SSFP sequence showing the importance of precise image planes when evaluating LVNC. Radial image planes with the placed through the centrepoint of the LV cavity avoids the risk of prescribing oblique planes. c Lateenhanced vertical long-axis sequence showed high signal throughout the trabeculae consistent with trabecular fibrosis (arrow) 
compaction. Most centres measure this parameter on SSFP sequences, and use high-resolution thin slices to do so (4$5 \mathrm{~mm}$ ). It is also useful to utilise radial slice projections, with the fulcrum passing through the centre of the LV (Fig. 11b). In this way, the risk of measuring the $\mathrm{NC} / \mathrm{C}$ ratio from an oblique slice is minimised. The diastolic ratio of $>2.3$ on CMR demonstrates high diagnostic accuracy in identifying pathological LVNC and is now widely regarded as the gold-standard.

Fibrosis may be observed in LVNC on CMR, which correlates with histological findings (Fig. 11c). Delayed enhancement is usually found in a subendocardial distribution. Delayed enhancement can also be found in some compacted normal segments indicating that fibrosis is also present in these normal appearing areas [89].

\section{Takotsubo cardiomyopathy}

Takotsubo cardiomyopathy (TTC) is also referred to as apical ballooning syndrome and stress cardiomyopathy. It was first described in Japan almost twenty years ago [92]. The diagnosis is based upon transient akinesia or dyskinesia of the LV apical, mid or basal segments extending beyond a single coronary vascular distribution; absence of angiographic evidence of acute plaque rupture or obstructive coronary artery disease; new ECG abnormalities or elevated troponin; no recent head trauma, intracranial bleeding, hypertrophic cardiomyopathy or phaeochromocytoma [93]. Takotsubo may also be accompanied by acute chest pain that when accompanied with ECG changes and cardiac enzyme rises explains why many patients are initially misdiagnosed with acute coronary syndrome.

The pathogenesis behind TTC has not been fully elucidated. Coronary spasm, coronary emboli with spontaneous fibrinolysis, abnormalities in the coronary microvascular function and regional myocarditis are some suggested etiologies. Numerous studies have linked it with catecholamine excess [94, 95]. One study described TTC in nine patients immediately following intravenous administration of epinephrine or dobutamine [95]. Both emotional and physical stresses are commonly seen as a precursor to TTC. Plasma catecholamine and neuropeptide levels in patients with TTC induced by emotional stress are markedly elevated compared with patients with myocardial infarction [94]. Endocardial biopsies of patients with TTC support excessive catecholamine exposure characterized by contraction bands, increased interstitial fibrosis and regional accumulation of inflammatory cells [96].

\section{CMR appearances}

The imaging appearances of TTC are currently evolving. Cine SSFP sequences in vertical long axis and short axis views allow the optimal assessment of left ventricular
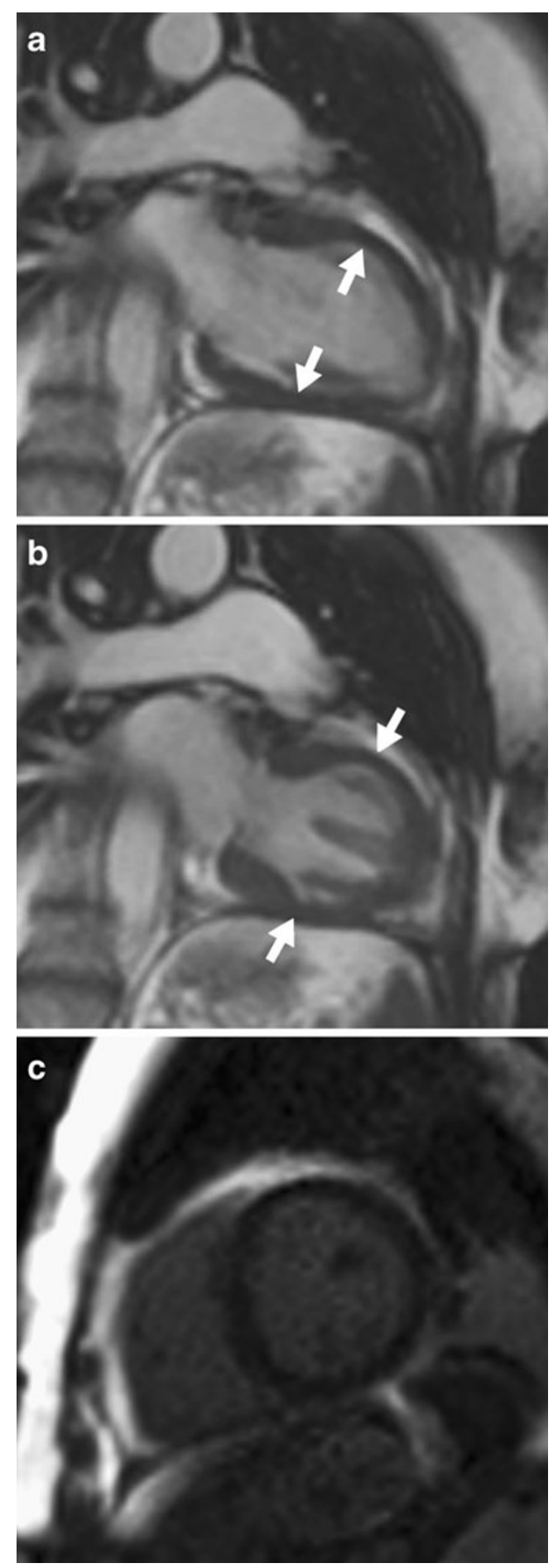

Fig. 12 A 54-year-old woman admitted with acute onset chest pain following a road traffic accident. Vertical long-axis SSFP sequence in (a) diastole and (b) systole showed akinesis of LV myocardial segments at the midventricular level. The basal and apical segments showed vigorous contraction. (c) Late-enhanced short-axis sequence showed an absence of scar, excluding significant infarction 
function, ventricular size and regional wall abnormalities. Several patterns of regional wall motion abnormalities have been described [95]. (1) Apical ballooning variant, which is characterized by apical akinesis with sparing of the base; (2) midventricular ballooning variant with midventricular akinesis with sparing of the apex and base (Fig. 12a, b); (3) basal ballooning variant with midventricular and basal akinesis with normal apical contractility. Right ventricular akinesia can also be seen in some patients. In this subgroup, it has been suggested that such patients may also have lower LV ejection fractions [97].

Eitel et al. [98] have highlighted the discriminating ability of CMR in patients presenting with acute chest pain syndromes. Late gadolinium enhancement (LGE) was seen in patients with myocardial infarctions and myocarditis, but not in patients with TTC (Fig. 12c). One study has demonstrated LGE in patients with TTC, though the LGE was of lower signal intensity than seen in myocardial infarction or myocarditis and the extent of tissue involvement was minimal [99].

Eitel et al. [98] evaluated a large group of patients presenting with TTC. Several interesting findings were described. Of 136 patients with TTC, 121 had significant stressful events $12 \mathrm{~h}$ preceding their TTC. Sixty-four patients had emotional stress and 57 had a physical stress. As with all other studies of TTC, post-menopausal women were more commonly affected, but $10 \%$ of patients were pre-menopausal, $10 \%$ were male and $10 \%$ did not recover LV function immediately [97].

\section{Conclusion}

CMR is an excellent imaging modality for the evaluation of the rare and unclassified cardiomyopathy. It allows the detection, morphology, function and myocardial contrastenhancement characteristics of these cardiomyopathy to be accurately and reproducibly analysed.

\section{References}

1. Rosamond W et al (2007) Heart disease and stroke statistics2007 update: a report from the American Heart Association statistics committee and stroke statistics subcommittee. Circulation 115(5):e69-e171

2. Quinones MA et al (2003) American College of Cardiology/ American Heart Association clinical competence statement on echocardiography: a report of the American College of Cardiology/ American Heart Association/American College of PhysiciansAmerican Society of Internal Medicine Task Force on clinical competenc. Circulation 107(7):1068-1089

3. Bluemke DA et al (2008) Noninvasive coronary artery imaging: magnetic resonance angiography and multidetector computed tomography angiography: a scientific statement from the american heart association committee on cardiovascular imaging and intervention of the council on cardiovascular radiology and intervention, and the councils on clinical cardiology and cardiovascular disease in the young. Circulation 118(5):586-606

4. Finn JP et al (2006) Cardiac MR imaging: state of the technology. Radiology 241(2):338-354

5. Pennell DJ et al (2004) Clinical indications for cardiovascular magnetic resonance (CMR): consensus panel report. Eur Heart J 25(21):1940-1965

6. Maron BJ et al (2006) Contemporary definitions and classification of the cardiomyopathies: an American Heart Association scientific statement from the council on clinical cardiology, heart failure and transplantation committee; quality of care and outcomes research and functional genomics and translational biology interdisciplinary working groups; and Council on Epidemiology and Prevention. Circulation 113(14):1807-1816

7. Elliott P et al (2008) Classification of the cardiomyopathies: a position statement from the European Society Of Cardiology Working Group on Myocardial and Pericardial Diseases. Eur Heart J 29(2):270-276

8. Selvanayagam JB et al (2007) Evaluation and management of the cardiac amyloidosis. J Am Coll Cardiol 50(22):2101-2110

9. Vanden Driesen RI, Slaughter RE, Strugnell WE (2006) MR findings in cardiac amyloidosis. AJR Am J Roentgenol 186 (6):1682-1685

10. Krombach GA et al (2007) Cardiac amyloidosis: MR imaging findings and $\mathrm{T} 1$ quantification, comparison with control subjects. J Magn Reson Imaging 25(6):1283-1287

11. Rathi VK et al (2008) Routine evaluation of left ventricular diastolic function by cardiovascular magnetic resonance: a practical approach. J Cardiovasc Magn Reson 10(1):36

12. Vogelsberg H et al (2008) Cardiovascular magnetic resonance in clinically suspected cardiac amyloidosis: noninvasive imaging compared to endomyocardial biopsy. J Am Coll Cardiol 51 (10): $1022-1030$

13. Syed IS et al (2010) Role of cardiac magnetic resonance imaging in the detection of cardiac amyloidosis. JACC Cardiovasc Imaging 3(2):155-164

14. Fattori R et al (1998) Contribution of magnetic resonance imaging in the differential diagnosis of cardiac amyloidosis and symmetric hypertrophic cardiomyopathy. Am Heart J 136(5): 824-830

15. Dutka DP et al (1999) Marked variation in the cardiomyopathy associated with Friedreich's ataxia. Heart 81(2):141-147

16. Durr A et al (1996) Clinical and genetic abnormalities in patients with Friedreich's ataxia. N Engl J Med 335(16):1169-1175

17. Harding AE (1981) Friedreich's ataxia: a clinical and genetic study of 90 families with an analysis of early diagnostic criteria and intrafamilial clustering of clinical features. Brain 104(3):589 620

18. Isnard $\mathrm{R}$ et al (1997) Correlation between left ventricular hypertrophy and GAA trinucleotide repeat length in Friedreich's ataxia. Circulation 95(9):2247-2249

19. Rajagopalan B et al (2010) Analysis of the factors influencing the cardiac phenotype in Friedreich's ataxia. Mov Disord 25(7):846-852

20. Meyer C et al (2007) Cardiomyopathy in Friedreich's ataxiaassessment by cardiac MRI. Mov Disord 22(11):1615-1622

21. Allanson JE (1987) Noonan syndrome. J Med Genet 24(1):9-13

22. Zenker M (2009) Genetic and pathogenetic aspects of Noonan syndrome and related disorders. Horm Res 72(Suppl 2):57-63

23. Zenker $\mathrm{M}$ et al (2007) SOS1 is the second most common Noonan gene but plays no major role in cardio-facio-cutaneous syndrome. J Med Genet 44(10):651-656

24. van der Burgt I (2007) Noonan syndrome. Orphanet J Rare Dis 2:4

25. Allanson JE et al (1985) Noonan syndrome: the changing phenotype. Am J Med Genet 21(3):507-514 
26. Shaw AC et al (2007) The natural history of Noonan syndrome: a long-term follow-up study. Arch Dis Child 92(2):128-132

27. Marino B et al (1999) Congenital heart diseases in children with Noonan syndrome: an expanded cardiac spectrum with high prevalence of atrioventricular canal. J Pediatr 135(6):703-706

28. Hudsmith LE et al (2006) Hypertrophic cardiomyopathy in Noonan Syndrome closely mimics familial hypertrophic cardiomyopathy due to sarcomeric mutations. Int J Cardiovasc Imaging 22(3-4):493-495

29. Danon MJ et al (1981) Lysosomal glycogen storage disease with normal acid maltase. Neurology 31(1):51-57

30. Sugie K et al (2002) Clinicopathological features of genetically confirmed Danon disease. Neurology 58(12):1773-1778

31. Yang $Z$ et al (2005) Danon disease as an underrecognized cause of hypertrophic cardiomyopathy in children. Circulation 112(11): $1612-1617$

32. van der Kooi AJ et al (2008) Extension of the clinical spectrum of Danon disease. Neurology 70(16):1358-1359

33. Charron P et al (2004) Danon's disease as a cause of hypertrophic cardiomyopathy: a systematic survey. Heart 90(8):842-846

34. Piotrowska-Kownacka D et al (2009) Cardiovascular magnetic resonance findings in a case of Danon disease. J Cardiovasc Magn Reson 11:12

35. Ntusi NB, Mayosi BM (2009) Aetiology and risk factors of peripartum cardiomyopathy: a systematic review. Int $\mathrm{J}$ Cardiol 131(2):168-179

36. Kawano $\mathrm{H}$ et al (2008) Magnetic resonance imaging in a patient with peripartum cardiomyopathy. Intern Med 47(2):97-102

37. Caballero-Borrego J et al (2008) Evidence of gadolinium lateenhancement on cardiac magnetic resonance imaging in a patient with peripartum cardiomyopathy. Rev Esp Cardiol 61(2):219-220

38. Leurent $G$ et al (2009) Contribution of cardiac MRI in the comprehension of peripartum cardiomyopathy pathogenesis. Int $\mathrm{J}$ Cardiol 132(3):e91-e93

39. Mouquet $\mathrm{F}$ et al (2008) Characterisation of peripartum cardiomyopathy by cardiac magnetic resonance imaging. Eur Radiol 18 (12):2765-2769

40. Marmursztejn J et al (2009) Delayed-enhanced cardiac magnetic resonance imaging features in peripartum cardiomyopathy. Int $\mathrm{J}$ Cardiol 137(3):e63-e64

41. Baruteau AE et al. (2009) Peripartum cardiomyopathy in the era of cardiac magnetic resonance imaging: first results and perspectives. Int J Cardiol Jan 24. [Epub ahead of print]

42. Emery AE (2002) The muscular dystrophies. Lancet 359 (9307):687-695

43. Nigro G et al (1990) The incidence and evolution of cardiomyopathy in Duchenne muscular dystrophy. Int J Cardiol 26(3):271-277

44. Yilmaz A et al (2008) Cardiac involvement in patients with Becker muscular dystrophy: new diagnostic and pathophysiological insights by a CMR approach. J Cardiovasc Magn Reson 10(1):50

45. Silva MC et al (2007) Myocardial delayed enhancement by magnetic resonance imaging in patients with muscular dystrophy. J Am Coll Cardiol 49(18):1874-1879

46. Pereira SR et al (2007) Association between a new 3q;5q chromosomal translocation and dystrophy of human retinal pigment epithelium. Genet Mol Res 6(4):1085-1090

47. Nigro G et al (1995) Evaluation of the cardiomyopathy in Becker muscular dystrophy. Muscle Nerve 18(3):283-291

48. Feldman AM, McNamara D (2000) Myocarditis. N Engl J Med 343(19):1388-1398

49. Leikin JB, Klein L (2000) Ephedra causes myocarditis. J Toxicol Clin Toxicol 38(3):353-354

50. Kurt TL (2000) Hypersensitivity myocarditis with ephedra use. J Toxicol Clin Toxicol 38(3):351

51. Zaacks SM et al (1999) Hypersensitivity myocarditis associated with ephedra use. J Toxicol Clin Toxicol 37(4):485-489
52. Naik SD, Freudenberger RS (2004) Ephedra-associated cardiomyopathy. Ann Pharmacother 38(3):400-403

53. Jacobs LJ (1989) Reversible dilated cardiomyopathy induced by methamphetamine. Clin Cardiol 12(12):725-727

54. Srikanth S, Barua R, Ambrose J (2008) Methamphetamineassociated acute left ventricular dysfunction: a variant of stressinduced cardiomyopathy. Cardiology 109(3):188-192

55. Lopez JE et al (2009) Recovery of methamphetamine associated cardiomyopathy predicted by late gadolinium enhanced cardiovascular magnetic resonance. J Cardiovasc Magn Reson 11(1):46

56. Wood DM et al. (2010) Recreational use of mephedrone (4methylmethcathinone, 4-MMC) with associated sympathomimetic toxicity. J Med Toxicol 6(3):327-330

57. Kmietowicz Z (2010) Home secretary bans mephedrone after taking advice from depleted council. BMJ 340:c1784

58. LeRoy EC et al (1988) Scleroderma (systemic sclerosis): classification, subsets and pathogenesis. J Rheumatol 15(2):202205

59. Takehara K, Soma Y, Ishibashi Y (1991) Early detection of scleroderma spectrum disorders in patients with Raynaud's phenomenon. Dermatologica 183(3):164-168

60. Poanta L et al (2009) Systolic and diastolic function in patients with systemic sclerosis. Eur J Intern Med 20(4):378-382

61. Kahan A, Allanore Y (2006) Primary myocardial involvement in systemic sclerosis. Rheumatology (Oxford) 45(Suppl 4):iv14-iv17

62. Tzelepis GE et al (2007) Pattern and distribution of myocardial fibrosis in systemic sclerosis: a delayed enhanced magnetic resonance imaging study. Arthritis Rheum 56(11):3827-3836

63. Weller PF, Bubley GJ (1994) The idiopathic hypereosinophilic syndrome. Blood 83(10):2759-2779

64. Bishop GG, Bergin JD, Kramer CM (2001) Hypereosinophilic syndrome and restrictive cardiomyopathy due to apical thrombi. Circulation 104(2):E3-E4

65. Salanitri GC (2005) Endomyocardial fibrosis and intracardiac thrombus occurring in idiopathic hypereosinophilic syndrome. AJR Am J Roentgenol 184(5):1432-1433

66. Goo HW, Han NJ, Lim TH (2001) Endomyocardial fibrosis mimicking right ventricular tumor. AJR Am J Roentgenol 177 (1):205-206

67. Syed IS et al (2008) Cardiac magnetic resonance imaging of eosinophilic endomyocardial disease. Int J Cardiol 126(3):e50 e52

68. Lam KY, Dickens P, Chan AC (1993) Tumors of the heart. A 20-year experience with a review of 12,485 consecutive autopsies. Arch Pathol Lab Med 117(10):1027-1031

69. Mukai K et al (1988) The incidence of secondary tumors of the heart and pericardium: a 10-year study. Jpn J Clin Oncol 18 (3):195-201

70. Klatt EC, Heitz DR (1990) Cardiac metastases. Cancer 65 (6): 1456-1459

71. Choe DH et al (1998) Obliteration of the pulmonary vein in lung cancer: significance in assessing local extent with CT. J Comput Assist Tomogr 22(4):587-591

72. Roberts WC, Glancy DL, DeVita VT Jr (1968) Heart in malignant lymphoma (Hodgkin's disease, lymphosarcoma, reticulum cell sarcoma and mycosis fungoides). A study of 196 autopsy cases. Am J Cardiol 22(1):85-107

73. Chiles $\mathrm{C}$ et al (2001) Metastatic involvement of the heart and pericardium: CT and MR imaging. Radiographics 21(2):439-449

74. Waller BF et al (1980) The "charcoal heart;" melanoma to the cor. Chest 77(5):671-676

75. Fujita N, Caputo GR, Higgins CB (1994) Diagnosis and characterization of intracardiac masses by magnetic resonance imaging. Am J Card Imaging 8(1):69-80

76. Rathi VK et al (2008) Cardiovascular magnetic resonance of the charcoal heart. J Cardiovasc Magn Reson 10(1):37 
77. Deetjen A et al (2006) Cardiac metastasis of a renal cell adenocarcinoma investigated by cardiac magnetic resonance imaging. Clin Res Cardiol 95(9):492-495

78. Oechslin EN et al (2000) Long-term follow-up of 34 adults with isolated left ventricular noncompaction: a distinct cardiomyopathy with poor prognosis. J Am Coll Cardiol 36(2):493-500

79. Chin TK et al (1990) Isolated noncompaction of left ventricular myocardium. A study of eight cases. Circulation 82(2):507-513

80. Sen-Chowdhry S, McKenna WJ (2008) Left ventricular noncompaction and cardiomyopathy: cause, contributor, or epiphenomenon? Curr Opin Cardiol 23(3):171-175

81. Petersen SE et al (2005) Left ventricular non-compaction: insights from cardiovascular magnetic resonance imaging. J Am Coll Cardiol 46(1):101-105

82. Jenni $\mathrm{R}$ et al (2001) Echocardiographic and pathoanatomical characteristics of isolated left ventricular non-compaction: a step towards classification as a distinct cardiomyopathy. Heart 86(6): 666-671

83. Burke A et al (2005) Left ventricular noncompaction: a pathological study of 14 cases. Hum Pathol 36(4):403-411

84. Borges AC, Kivelitz D, Baumann G (2003) Isolated left ventricular non-compaction: cardiomyopathy with homogeneous transmural and heterogeneous segmental perfusion. Heart 89(8):e21

85. Finsterer J, Stollberger C, Kopsa W (2005) Multidisciplinary diagnostic approach for left ventricular hypertrabeculation/noncompaction. Yonsei Med J 46(2):309-310, author reply 310-2

86. Lilje C et al (2006) Complications of non-compaction of the left ventricular myocardium in a paediatric population: a prospective study. Eur Heart J 27(15):1855-1860

87. Bagur RH et al (2008) Images in cardiovascular medicine. Ebstein anomaly associated with left ventricular noncompaction. Circulation 118(16):e662-e664

88. Pignatelli RH et al (2003) Clinical characterization of left ventricular noncompaction in children: a relatively common form of cardiomyopathy. Circulation 108(21):2672-2678
89. Dursun $M$ et al (2010) MR imaging features of ventricular noncompaction: emphasis on distribution and pattern of fibrosis. Eur J Radiol 74(1):147-151

90. Kohli SK et al (2008) Diagnosis of left-ventricular noncompaction in patients with left-ventricular systolic dysfunction: time for a reappraisal of diagnostic criteria? Eur Heart J 29(1):8995

91. Cerqueira MD et al (2002) Standardized myocardial segmentation and nomenclature for tomographic imaging of the heart: a statement for healthcare professionals from the Cardiac Imaging Committee of the Council on Clinical Cardiology of the American Heart Association. Circulation 105(4):539-542

92. Dote K et al (1991) Myocardial stunning due to simultaneous multivessel coronary spasms: a review of 5 cases. J Cardiol 21(2):203-214

93. Eitel I et al (2010) Inflammation in takotsubo cardiomyopathy: insights from cardiovascular magnetic resonance imaging. Eur Radiol 20(2):422-431

94. Wittstein IS et al (2005) Neurohumoral features of myocardial stunning due to sudden emotional stress. N Engl J Med 352 (6):539-548

95. Abraham J et al (2009) Stress cardiomyopathy after intravenous administration of catecholamines and beta-receptor agonists. J Am Coll Cardiol 53(15):1320-1325

96. Nef HM et al (2007) Tako-Tsubo cardiomyopathy: intraindividual structural analysis in the acute phase and after functional recovery. Eur Heart J 28(20):2456-2464

97. Sharkey SW et al (2010) Natural history and expansive clinical profile of stress (tako-tsubo) cardiomyopathy. J Am Coll Cardiol 55(4):333-341

98. Eitel I et al (2008) Differential diagnosis of suspected apical ballooning syndrome using contrast-enhanced magnetic resonance imaging. Eur Heart J 29(21):2651-2659

99. Rolf A et al (2009) Immunohistological basis of the late gadolinium enhancement phenomenon in tako-tsubo cardiomyopathy. Eur Heart J 30(13):1635-1642 